\title{
Analysis of the viscoelastic behavior of plates made of functionally graded materials
}

\author{
Holm Altenbach ${ }^{1, *}$ and Victor A. Eremeyev ${ }^{2, * *}$ \\ ${ }^{1}$ Lehrstuhl für Technische Mechanik, Zentrum für Ingenieurwissenschaften, Martin-Luther-Universität Halle-Wittenberg, \\ 06099 Halle (Saale), Germany \\ ${ }^{2}$ South Scientific Center of RASci and South Federal University, 8a, Milchakov St, 344090 Rostov on Don, Russia
}

Key words Viscoelasticity, plates, direct approach, polymer foams, effective stiffness, functionally graded materials.

Dedicated to our colleague and friend Peter Haupt on the occasion of his 70 th birthday.

Considering the viscoelastic behavior of polymer foams, a new plate theory based on the direct approach is introduced and applied to plates composed of functionally graded materials (FGM). The governing two-dimensional equations are formulated for a deformable surface and the stiffness parameters are identified for the linear viscoelastic isotropic material behavior. It is assumed that the material properties are changing in thickness direction. Solving the plate bending problem of the global mechanical analysis, it will be demonstrated that in some cases, the results significantly differ from the results based on the classical Kirchhoff-type theory.

\section{Introduction}

Mechanics of materials is one of the oldest branches of mechanics but it is of current and emerging interest of physicians, materials scientists, and engineers. The concepts of continuum mechanics and mechanics of materials are summarized in a huge number of textbooks and monographs. As an example let us note here only the book written by Peter Haupt [1]. The reason for the continuous development of new theories within the mechanics of materials firstly one has an increasing number of new materials (for example, nanomaterials, foams, biomaterials, shape memory alloys and polymers, smart materials, etc.), and secondly the necessity to take into account new complex effects of the material behavior. The structural analysis of elements made of such new materials is based not only on the geometrical description and the static equilibrium or equation of motion. In addition one needs an accurate and physically based material behavior description substituting the more or less empirical (and in many cases very sophisticated) sets of constitutive equations.

One example of a very prospective class of new materials are foams. Metallic and polymeric foams are more and more used as a material for lightweight structures [2-6]. Such structures are applied in the automotive or airspace industries since they combine low weight, high specific strength, and excellent possibilities to absorb energy. The technical realization is mostly performed as sandwich panels (plates or shells with hard and stiff face sheets and a core layer made of a foam). From the mechanical point of view, a foam is a very complex material which demonstrates many non-classical properties (for example, some foams have a negative Poisson ratio). The foam itself can be modeled as a functionally graded material with mechanical properties changing over the thickness direction. There are two types of metal or polymer foams. One is the closed-cell foam, while the second one is the open-cell foam. The defining characteristic of the foams is the very high porosity: typically well over $80 \%, 90 \%$, and even $98 \%$ of the volume consists of void spaces. Examples of closed-cell foams with different porosity are presented in Fig. 1.

The analysis of plates and shell structures can be performed on the base of different theories. Finally, all theories are presented by two-dimensional field equations, but the ways to deduce these equations are not unique. Mostly engineering hypotheses on the stress, strain, or displacement states in the plate are used leading to some simplifications of the basic three-dimensional equations. They result in two-dimensional equations connecting displacements and rotations with strains by kinematical equations, stating the static equilibrium or equation of motion and establishing the constitutive relations. In a similar way, by applying some mathematical techniques like the power series expansion [8] or the asymptotic integration [9] one can observe the set of two-dimensional governing equations. Note that both approaches have a unique starting point

* Corresponding author, e-mail: holm.altenbach@iw.uni-halle.de

** e-mail: eremeyev@math.rsu.ru 

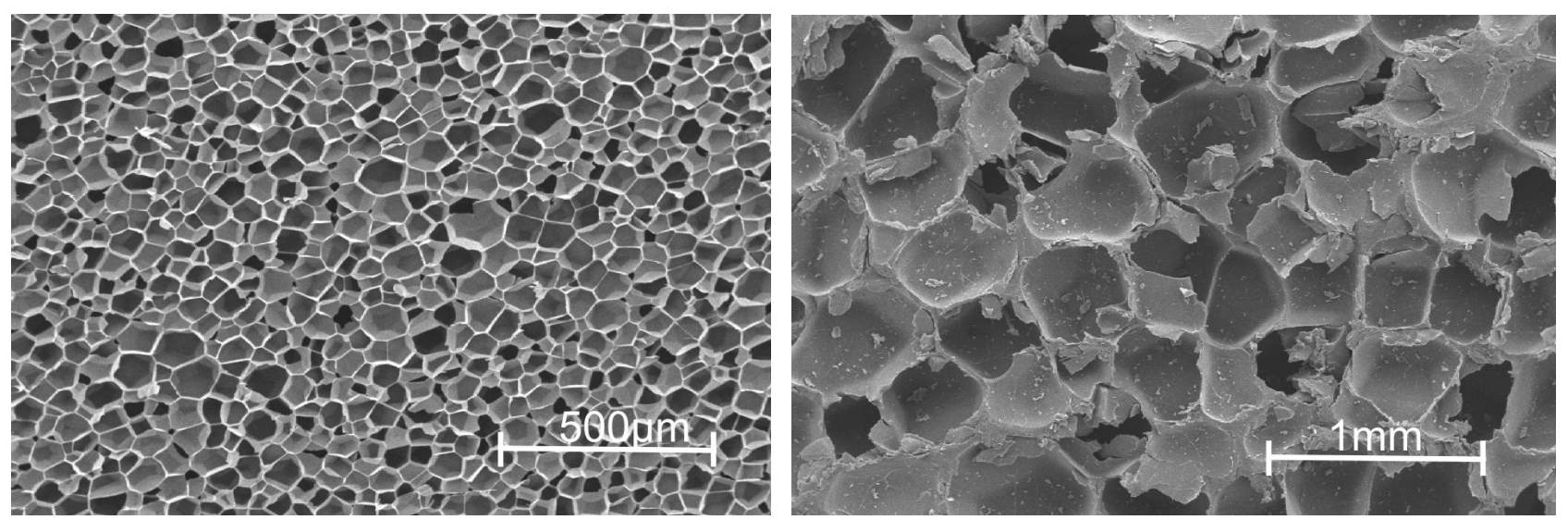

Fig. 1 Polymer closed-cell foams with different density (photos by curtesy of A. Kraatz, German Institute for Polymers, Darmstadt [7])

- the equations for the three-dimensional continuum. In contrast, the direct approach is based on the straight-forward introduction of the two-dimensional equations. This approach in combination with the effective properties concept allows the global analysis of all branches of plate theories (homogeneous, sandwich, laminated, etc.). The different possibilities of the formulation of plate theories are discussed in [10-12] among others.

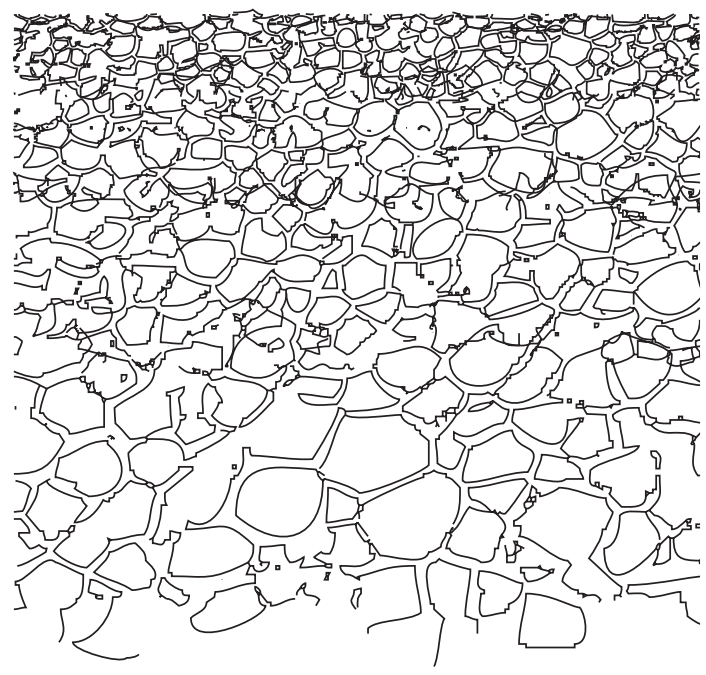

Fig. 2 Nonhomogeneous structure of the foam

In this contribution, we present a new theory based on the direct approach combined with the effective properties concept. We consider plates made of polymer foams with a highly nonhomogeneous structure through the thickness (see Fig. 2) and apply the theory of plates and shells formulated in [13-17]. From the direct approach point of view, a plate or a shell is modeled as a material surface each particle of which has five degrees of freedom (three displacements and two rotations, the rotation about the normal to the plate is not considered). Such a model can be accepted in the case of plates with constant or slow changing thickness. For the linear elastic variant the identification of the elastic stiffness tensors was proposed in [18-20]. Using the techniques presented in these articles the static boundary-value problems for FGM plates made of metal foams which behave elastically are solved in [21]. Here we extend this analysis to the case of viscoelastic polymer foams.

\section{Governing equations}

Let us consider for simplicity the geometrically and physically linear theory. In addition, we assume plate-like structures. The basic equations connecting the strains with the displacements and rotations or stating the static equilibrium or the equation of motion can be deduced by applying hypotheses (like the Kirchhoff's hypotheses) or mathematical techniques (like power series expansion). In both cases one gets the expressions for the constitutive behavior assuming elastic or inelastic material behavior. 
A quite different way is given by the direct approach. The starting point in this case is a two-dimensional deformable surface. On each part of this deformable surface forces and moments are acting - they are the primary variables. The next step is the introduction of the deformation measures. Finally, it is necessary to combine the forces and the moments with the deformation variables (constitutive equations). But the identification of the effective properties (stiffness and other parameters) must be performed for each class of plates individually, solving, for example, boundary value problems. The identification of the two-dimensional characteristics is a non-trivial problem since they must be computed from the threedimensional parameters applying assumptions like the introduction of stress resultants (forces and moments) instead the stress tensor components.

Let us introduce the governing equations. The equations of motion are formulated as the Euler's laws of dynamics [19-21]

$$
\nabla \cdot \boldsymbol{T}+q=\rho \ddot{u}+\rho \Theta_{1} \cdot \ddot{\varphi}, \quad \nabla \cdot M+T_{\times}+m=\rho \Theta_{1}^{\mathrm{T}} \cdot \ddot{u}+\rho \boldsymbol{\Theta}_{2} \cdot \ddot{\varphi} .
$$

Here $T, M$ are the tensors of forces and moments, $\boldsymbol{q}, m$ are the surface loads (forces and moments), $\boldsymbol{T}_{\times}$is the vector invariant of the force tensor [22], $\nabla$ is the nabla (Hamilton) operator, $\boldsymbol{u}, \boldsymbol{\varphi}$ are the vectors of displacements and rotations, $\Theta_{1}, \Theta_{2}$ are the first and the second tensor of inertia, $\rho$ is the density (effective property of the deformable surface), $(\ldots)^{\mathrm{T}}$ denotes transposed and $(\ldots)$ is the time derivative. The geometrical equations are given as

$$
\mu=(\nabla u \cdot a)^{\mathrm{sym}}, \quad \gamma=\nabla u \cdot n+c \cdot \varphi, \quad \kappa=\nabla \varphi .
$$

$a$ is the first metric tensor (plane tensor), $n$ is the unit outer normal vector at the surface, $c=-a \times n$ is the discriminant tensor, $\mu, \gamma$, and $\kappa$ are the strain tensors (the tensor of in-plane strains, the vector of transverse shear strains, and the tensor of the out-of-plane strains, respectively), $(\ldots)^{\text {sym }}$ denotes the symmetric part.

The boundary conditions are given by the relations

$$
\nu \cdot T=f, \quad \nu \cdot M=l \quad(l \cdot n=0) \quad \text { or } \quad u=u^{0}, \quad \varphi=\varphi^{0} \quad \text { along } S .
$$

Here $f$ and $l$ are external force and moment vectors, respectively, acting along the boundary $S$ of the plate, while $u^{0}$ and $\varphi^{0}$ are given functions describing the displacements and rotations of the plate boundary, respectively. $\nu$ is the unit outward normal vector in the tangential plane to the boundary $S(\nu \cdot \boldsymbol{n}=0)$. The relations (2) are the static and the kinematic boundary conditions. Other types of boundary conditions are possible. For example, the boundary conditions corresponding to a hinge are given by

$$
\nu \cdot M \cdot \tau=0, \quad u=0, \quad \varphi \cdot \tau=0 .
$$

Here, $\tau$ is the unit tangent vector in the tangential plane to the boundary $S(\tau \cdot n=\tau \cdot \nu=0)$.

Polymers near their glass transition temperature behave like viscoelastic materials. That means that the moduli of the polymers depend on the strain-rate or the time of loading. Thus, a foam made of such a polymer behaves viscoelastically too. The two-dimensional constitutive equations of a viscoelastic plate were formulated in the general form in [15]. For simplicity, let us consider a through-the-thickness symmetric structure of the plate under consideration and an isotropic material behavior. In this case, the constitutive equations for the stress resultants follows as:

- in-plane forces

$$
T \cdot \boldsymbol{a}=\mathcal{A} \mu \equiv \int_{-\infty}^{t} A(t-\tau) \cdot \cdot \dot{\mu} \mathrm{d} \tau,
$$

- transverse shear forces

$$
T \cdot \boldsymbol{n}=\mathcal{G} \gamma \equiv \int_{-\infty}^{t} \Gamma(t-\tau) \cdot \dot{\gamma} \mathrm{d} \tau
$$

- moments

$$
M^{\mathrm{T}}=\mathcal{C} \kappa \equiv \int_{-\infty}^{t} C(t-\tau) \cdot \cdot \dot{\kappa} \mathrm{d} \tau .
$$


Here, $\mathcal{A}, \mathcal{G}$, and $\mathcal{C}$ are linear viscoelastic operators, $A(t), C(t)$ are 4 th rank tensors, $\Gamma(t)$ is a 2 nd rank tensor which describes the effective stiffness properties (relaxation functions for the plate). They depend on the material properties and the crosssection geometry. In the case of isotropic and symmetric-over-the-thickness plates the effective stiffness tensors have the following structure [17]

$$
A=A_{11} a_{1} a_{1}+A_{22}\left(a_{2} a_{2}+a_{4} a_{4}\right), \quad C=C_{22}\left(a_{2} a_{2}+a_{4} a_{4}\right)+C_{33} a_{3} a_{3}, \quad \Gamma=\Gamma \boldsymbol{a},
$$

with

$$
a_{1}=a=e_{1} e_{1}+e_{2} e_{2}, \quad a_{2}=e_{1} e_{1}-e_{2} e_{2}, \quad a_{3}=c=e_{1} e_{2}-e_{2} e_{1}, \quad a_{4}=e_{1} e_{2}+e_{2} e_{1} .
$$

$\boldsymbol{e}_{1}, \boldsymbol{e}_{2}$ are the unit basic vectors. In addition, one obtains the orthogonality condition for the $\boldsymbol{a}_{i}(i=1,2,3,4)$

$$
\frac{1}{2} a_{i} \cdot a_{j}=\delta_{i j}
$$

where $\delta_{i j}$ is the Kronecker's symbol.

\section{Effective properties}

For elastic plates, the identification of the components of the effective stiffness tensors was shown in [17-20]. By the same technique, the analogous viscoelastic stiffness tensor components can be computed [15]. Below we discuss the special case of the standard viscoelastic body.

Let us consider the three-dimensional isotropic viscoelastic constitutive equations [1,23]

$$
\sigma=\int_{-\infty}^{t} 2 \mu(t-\tau) \dot{e} \mathrm{~d} \tau+\int_{-\infty}^{t} K(t-\tau) \dot{e} I \mathrm{~d} \tau, \quad e=\frac{1}{3} \operatorname{tr} \varepsilon, \quad e=\varepsilon-e I
$$

or

$$
\varepsilon=\int_{-\infty}^{t} M(t-\tau) \dot{\boldsymbol{s}} \mathrm{d} \tau+\int_{-\infty}^{t} J(t-\tau) \dot{\sigma} \boldsymbol{I} \mathrm{d} \tau, \quad \sigma=\frac{1}{3} \operatorname{tr} \boldsymbol{\sigma}, \quad \boldsymbol{s}=\boldsymbol{\sigma}-\sigma \boldsymbol{I},
$$

where $\sigma$ and $\varepsilon$ are the stress and the strain tensor, respectively, $\boldsymbol{I}$ is the three-dimensional unit tensor. $\mu(t)$ and $K(t)$ are the shear and the bulk relaxation functions, while $M(t)$ and $J(t)$ are the shear and the bulk creep functions. Alternative expressions (but not equivalent!) for the integral constitutive equations of a viscoelastic body are the differential constitutive equations [23]

$$
P\left(\partial_{t}\right) \sigma=Q\left(\partial_{t}\right) \varepsilon, \quad P_{1}\left(\partial_{t}\right) \boldsymbol{s}=Q_{1}\left(\partial_{t}\right) \boldsymbol{e}
$$

where $\partial_{t}(\ldots)$ denotes the time derivative, $P\left(\partial_{t}\right), P_{1}\left(\partial_{t}\right), Q\left(\partial_{t}\right), Q_{1}\left(\partial_{t}\right)$ are polynomials. In addition, there exists a third type of expressions - the complex moduli representation.

A special case of (9) is the standard linear viscoelastic body [1]

$$
\dot{\sigma}+\frac{\hat{K}}{\eta} \sigma=\frac{K_{\infty} \hat{K}}{\eta} \varepsilon+\left(K_{\infty}+\hat{K}\right) \dot{\varepsilon}, \quad \dot{s}+\frac{\hat{G}}{\eta_{1}} s=\frac{G_{\infty} \hat{G}}{\eta_{1}} e+\left(G_{\infty}+\hat{G}\right) \dot{e} .
$$

Here $\hat{K}$ and $K_{\infty}$ are the instantaneous and long-term bulk moduli, $\hat{G}$ and $G_{\infty}$ are the instantaneous and long-term shear moduli, and $\eta$ and $\eta_{1}$ are the viscosities at the hydrostatic and the shear loadings, respectively. All these properties should be estimated experimentally.

Due to (10), it yields that the following relations are valid [1]

$$
K(t)=K_{\infty}+\hat{K} \exp \left(-\frac{\hat{K}}{\eta} t\right), \quad \mu(t)=G_{\infty}+\hat{G} \exp \left(-\frac{\hat{G}}{\eta_{1}} t\right) .
$$

Using the Laplace transform of a function $f(t)$

$$
\bar{f}(s)=\int_{0}^{\infty} f(t) e^{-s t} \mathrm{~d} t
$$


one can write Eqs. (7), (8) in the form [1]

$$
\bar{\sigma}=2 s \mu(s) \overline{\boldsymbol{e}}+s K(s) \bar{e} \boldsymbol{I}, \quad \bar{\varepsilon}=s M(s) \overline{\boldsymbol{s}}+s J(s) \bar{\sigma} \boldsymbol{I} .
$$

Further we consider two cases:

Case 1: Homogeneous plates - all properties are constant (no dependency on the thickness coordinate $z$ ).

Case 2: Inhomogeneous plates (sandwich, multilayered, functionally graded) - all properties are even functions of $z$.

Note that in both cases we have no coupling between the in-plane and the out-of-plane behavior.

In the case of isotropic material behavior one has two material properties describing the viscoelastic behavior. They depend on the thickness coordinate $z$ and on the time $t$

$$
K=K(z, t), \quad \mu=\mu(z, t)
$$

In addition, a density function must be considered. Let us assume the simplest case - the density depends only on the thickness coordinate

$$
\rho_{0}=\rho_{0}(z)
$$

$\rho_{0}$ is the density of the three-dimensional solid.

Using the analogy between (12) and the Hooke's law we can extend the identification procedure [17-20] to the Laplace mapping of the effective relaxation or creep functions, see [15]. The in-plane (membrane) stiffness tensor components are

$$
\left.\bar{A}_{11}=\frac{1}{2}\left\langle\frac{\bar{E}}{1-\bar{\nu}}\right\rangle, \quad \bar{A}_{22}=\frac{1}{2}\left\langle\frac{\bar{E}}{1+\bar{\nu}}\right\rangle=<\bar{\mu}\right\rangle
$$

the out-of-plane (plate) stiffness tensor components are

$$
\bar{C}_{33}=\frac{1}{2}\left\langle\frac{\bar{E}}{1-\bar{\nu}} z^{2}\right\rangle, \quad \bar{C}_{22}=\frac{1}{2}\left\langle\frac{\bar{E}}{1+\bar{\nu}} z^{2}\right\rangle=<\bar{\mu} z^{2}>,
$$

and the transverse shear stiffness tensor component is

$$
\bar{\Gamma}=\lambda^{2} \bar{C}_{22}
$$

with $\lambda$ following from a Sturm-Liouville problem

$$
\frac{\mathrm{d}}{\mathrm{d} z}\left(\bar{\mu} \frac{\mathrm{d} Z}{\mathrm{~d} z}\right)+\lambda^{2} \bar{\mu} Z=0,\left.\quad \frac{\mathrm{d} Z}{\mathrm{~d} z}\right|_{|z|=\frac{h}{2}}=0 .
$$

Here $<(\ldots)>=\int_{-h / 2}^{h / 2}(\ldots) \mathrm{d} z, h$ is the thickness of the plate. The following relations hold true [23]

$$
\bar{E}=\frac{9 \bar{\mu} \bar{K}}{\bar{\mu}+3 \bar{K}}, \quad \bar{\nu}=\frac{3 \bar{K}-2 \bar{\mu}}{2(\bar{\mu}+3 \bar{K})} .
$$

The corresponding relaxation functions $E(t)$ and $\nu(t)$ may be used instead of $\mu(t)$ and $K(t)$. Let us note that for the viscoelastic plate $\bar{\mu}=\bar{\mu}(z, s)$. Thus, $\lambda=\lambda(s)$. The tensors of inertia and the plate density are given by $[14,17]$

$$
\rho=\left\langle\rho_{0}\right\rangle, \quad \rho \Theta_{1}=-\left\langle\rho_{0} z\right\rangle c, \quad \rho \Theta_{2}=\Theta a, \quad \Theta=\left\langle\rho_{0} z^{2}\right\rangle .
$$

Considering the symmetry of the thickness geometry and of the material properties of the plate, from (17) one gets that $\Theta_{1}=\mathbf{0} . \Theta$ characterizes the rotatory inertia of the cross-section of the plate. 


\section{Examples of effective stiffness relaxation functions}

For the sake of simplicity, let us consider the case $\nu(t)=\nu=$ const (see [23] for details). That means that the following relations hold true

$$
E(t)=2 \mu(t)(1+\nu), \quad K(t)=\frac{2 \mu(t)(1+\nu)}{3(1-2 \nu)} .
$$

For the constitutive equations of the standard viscoelastic solid, the latter relation implies that $\eta_{1}=\eta$, and

$$
K_{\infty}=\frac{2 G_{\infty}(1+\nu)}{3(1-2 \nu)}, \quad \hat{K}=\frac{2 \hat{G}(1+\nu)}{3(1-2 \nu)} .
$$

Thus, in this case one gets four independent material constants. They are $G_{\infty}, \hat{G}$ (or $K_{\infty}, \hat{K}$ ), $\eta$, and $\nu$.

\subsection{Homogeneous plate}

The simplest test for the correctness of the estimated stiffness properties is the homogeneous isotropic plate. The basic geometrical property is the thickness $h$, the material properties of the plate are symmetric with respect to the mid-plane. All material properties are constant over the thickness, that means they do not depend on the thickness coordinate. The non-zero components of the classical relaxation tensors are

$$
\begin{aligned}
A_{11}(t) & =\frac{E(t) h}{2(1-\nu)}, \quad A_{22}(t)=\frac{E(t) h}{2(1+\nu)}=\mu(t) h, \\
C_{33}(t) & =\frac{E(t) h^{3}}{24(1-\nu)}, \quad C_{22}(t)=\frac{E(t) h^{3}}{24(1+\nu)}=\frac{\mu(t) h^{3}}{12} .
\end{aligned}
$$

The density and the rotatory inertia coefficient are

$$
\rho=\rho_{0} h, \quad \Theta=\frac{\rho_{0} h^{3}}{12} .
$$

The transverse shear relaxation function follows from (15). The solution of (16) with $\bar{\mu}=\bar{\mu}(s)$ is given by $\cos \lambda z=0$ with its smallest eigenvalue

$$
\lambda=\frac{\pi}{h},
$$

which does not depend on $s$. Finally, one obtains

$$
\Gamma(t)=\frac{\pi^{2}}{h^{2}} \frac{\mu(t) h^{3}}{12}=\frac{\pi^{2}}{12} \mu(t) h .
$$

$\pi^{2} / 12$ is the so-called shear correction factor which was first introduced by Timoshenko [24] in the theory of beams. The value coincides with the Mindlin's estimate in the plate theory [25] from which Reissner's estimate 5/6 [26] differs slightly.

It is evident that in the case of homogeneous viscoelastic plates with constant Poisson ratio one gets the same relations for the effective stiffness tensors as in the case of elastic plates $[19,20]$. There is only one difference - they are now functions of $t$.

\subsection{Functionally graded material}

In this paragraph we consider small deformations of a functionally graded plate made of a viscoelastic polymer foam. For the strip made of a porous polymer foam the distribution of the pores over the thickness can vary significantly (see, for example, Fig. 2). Let us introduce $h$ as the thickness of the panel, $\rho_{\mathrm{s}}$ as the density of the bulk material and $\rho_{\mathrm{p}}$ as the minimum value of the density of the foam. For the description of the symmetric distribution of the porosity we assume the power law [21]

$$
V(z)=\alpha+(1-\alpha)\left|\frac{2 z}{h}\right|^{n}
$$


where

$$
\alpha=\frac{\rho_{\mathrm{p}}}{\rho_{\mathrm{s}}}
$$

is the minimal relative density. $n=0$ corresponds to the homogeneous plate described in the previous paragraph.

The properties of a foam strongly depend on the porosity and the cell structure. For the polymer foam, in [5] the modification of the standard linear viscoelastic solid is proposed. For the open-cell foam, the constitutive law has the form

$$
\dot{\sigma}+\frac{\hat{K}}{\eta} \sigma=C_{1} V(z)^{2}\left[\frac{K_{\infty} \hat{K}}{\eta} \varepsilon+\left(K_{\infty}+\hat{K}\right) \dot{\varepsilon}\right]
$$

while for the closed-cell foam the constitutive equation has the form

$$
\dot{\sigma}+\frac{\hat{K}}{\eta} \sigma=C_{2}\left[\phi^{2} V(z)^{2}+(1-\phi) V(z)\right]\left[\frac{K_{\infty} \hat{K}}{\eta} \varepsilon+\left(K_{\infty}+\hat{K}\right) \dot{\varepsilon}\right] .
$$

Here, $C_{1} \approx 1, C_{2} \approx 1$, and $\phi$ describe the relative volume of the solid polymer concentrated near the cell edges. Usually, $\phi=0.6 \ldots 0.7 . K_{\infty}, \hat{K}, \eta$ are material constants of the polymer used in manufacturing of the foam.

From Eqs. (21), (22) one can see that the corresponding relaxation functions are given by the relations

$$
K=K(z, t)=K(t) \kappa(z)
$$

where $K(t)$ is defined by Eq. (11), while $\kappa(z)=C_{1} V(z)^{2}$ for the open-cell foam and $\kappa(z)=C_{2}\left[\phi^{2} V(z)^{2}+(1-\phi) V(z)\right]$ for the closed-cell foam, respectively. By analogy to (23), the following relation can be established for the shear relaxation function

$$
\mu=\mu(z, t)=\mu(t) m(z)
$$

Eqs. (23) and (24) have the meaning that the viscoelastic properties of a foam, for example, the time of relaxation, do not depend on the porosity distribution. Note, that representations (23) and (24) are only simplifying assumptions for spatial nonhomogeneous foams.

Using experimental data presented in $[2,5]$ one can assume $\nu=$ const. In this case, we obtain that $A_{11}, A_{22}, C_{33}, C_{22}$ are connected by

$$
A_{11}=\frac{1+\nu}{1-\nu} A_{22}, \quad C_{33}=\frac{1+\nu}{1-\nu} C_{22} .
$$

For the open-cell foam $A_{22}$ and $C_{22}$ are given by

$$
A_{22}=h\left[\alpha^{2}+\frac{2 \alpha(1-\alpha)}{n+1}+\frac{(1-\alpha)^{2}}{2 n+1}\right] \mu(t), \quad C_{22}=\frac{h^{3}}{12}\left[\alpha^{2}+\frac{6 \alpha(1-\alpha)}{n+3}+\frac{3(1-\alpha)^{2}}{2 n+3}\right] \mu(t)
$$

while for the closed-cell foam by

$$
\begin{aligned}
& A_{22}=h\left\{\phi^{2}\left[\alpha^{2}+\frac{2 \alpha(1-\alpha)}{n+1}+\frac{(1-\alpha)^{2}}{2 n+1}\right]+(1-\phi)\left[\alpha+\frac{1-\alpha}{n+1}\right]\right\} \mu(t), \\
& C_{22}=\frac{h^{3}}{12}\left\{\phi^{2}\left[\alpha^{2}+\frac{6 \alpha(1-\alpha)}{n+3}+\frac{3(1-\alpha)^{2}}{2 n+3}\right]+(1-\phi)\left[\alpha+\frac{3(1-\alpha)}{n+3}\right]\right\} \mu(t) .
\end{aligned}
$$

Here it was assumed that $C_{1}=1, C_{2}=1$, and that $\phi$ does not depend on $z$.

From Eqs. (26), (27) it is easy to see that the classical relaxation functions differ only by factors from the shear relaxation function. Note, that one can easily extend Eqs. (21), (22) to the case of general constitutive equations (7) or (9). Thus, using the assumption that $\nu=$ const, one can calculate the classical effective stiffness relaxation functions for the general viscoelastic constitutive equations multiplying the shear relaxation function with the corresponding factor similar to Eqs. (26), (27). In the more general situation with $\nu=\nu(t)$ or taking into account other viscoelastic phenomena, for example, the filtration of a fluid in the saturated foam, the effective stiffness relaxation functions may be more complex than for the pure solid polymer discussed here. 
To obtain the dependence of the transverse shear stiffness relaxation function we have to solve Eq. (16). In the general case, the solution of the spectral problem (16) may be performed numerically only. For example, in [21] the shooting method [27] was used. Let us note that for the viscoelastic plate $\bar{\mu}=\bar{\mu}(z, s)$. Thus, $\lambda=\lambda(s)$. It means that for the determination of $\Gamma(t)$ one has to solve (16) for any arbitrary value of $s$ and with the help of $\lambda=\lambda(s)$ to find numerically the inverse Laplace transform of $\lambda^{2}(s) \bar{C}_{22}(s)$. But in the special case of Eq. (24) one gets that $\bar{\mu}=\bar{\mu}(s) m(z)$. That means that $\lambda$ does not depend on $s$, and thus $\Gamma(t)=\lambda^{2} C_{22}(t)$. For the sake of simplicity, we will further take assumption (24) into account.

Let us find the bounds for the values of $\lambda$. Introducing a new independent variable $\zeta$ by the formula

$$
\zeta=\int_{-h / 2}^{z} \frac{\mathrm{d} z}{m(z)}
$$

one can transform (16) to the form (for details see, for example, [28])

$$
\frac{\mathrm{d}^{2} Z}{\mathrm{~d} \zeta^{2}}+\lambda^{2} m^{2} Z=0,\left.\quad \frac{\mathrm{d} Z}{\mathrm{~d} \zeta}\right|_{\zeta=0, L}=0
$$

Here $L$ denotes

$$
L=\int_{-h / 2}^{h / 2} \frac{\mathrm{d} z}{m(z)}
$$

Substituting $\zeta=\zeta / L$, one can transform the spectral problem (28) to the canonical form

$$
\frac{\mathrm{d}^{2} Z}{\mathrm{~d} \zeta^{2}}+\lambda^{2} L^{2} m^{2} Z=0,\left.\quad \frac{\mathrm{d} Z}{\mathrm{~d} \zeta}\right|_{\zeta=0,1}=0
$$

The following theorem holds [29]:

Theorem 3.1. If one has two eigen-value problems

$$
\frac{\mathrm{d}^{2} Z}{\mathrm{~d} \zeta^{2}}+\lambda^{2} f_{1} Z=0, \quad \frac{\mathrm{d}^{2} Z}{\mathrm{~d} \zeta^{2}}+\lambda^{2} f_{2} Z=0,\left.\quad \frac{\mathrm{d} Z}{\mathrm{~d} \zeta}\right|_{\zeta=0,1}=0
$$

with two functions $f_{1}(\zeta)$ and $f_{2}(\zeta)$ such that $f_{1} \leq f_{2}$, then the following inequality holds true $\lambda_{1} \geq \lambda_{2}$. Here $\lambda_{1}$ and $\lambda_{2}$ are the eigen-values corresponding to the functions $f_{1}(\zeta)$ and $f_{2}(\zeta)$, respectively.

Using this theorem and the inequality $m_{\min } \leq m \leq m_{\max }$, we obtain the lower and upper bounds of $\lambda$

$$
\frac{\pi}{L m_{\max }} \leq \lambda \leq \frac{\pi}{L m_{\min }}
$$

For the homogeneous plate $m_{\min }=m_{\max }=m, L=h / m$, and both bounds coincide.

Finally, we should mention that in the case of constant Poisson's ratio and with the assumption (24) the determination of the effective in-plane, bending, and transverse shear stiffness tensors of a symmetric FGM viscoelastic plate made of a polymer foam can be computed by the same method as for elastic plates [21]. The relaxation functions for viscoelastic FGM plates can be found from the values of the corresponding effective stiffness of an elastic FGM plate by multiplication with the normalized shear relaxation function of the polymer solid.

\section{Viscoelastic bending behavior of a plate made of functionally graded material}

Considering the symmetry of the material properties with respect to the mid-plane one gets a decoupling of the in-plane and the plate states. Let us assume the plate bending problem with $m=0$. Using the results presented in [21] and the Laplace transform, one can reduce the set of governing equations to

$$
\bar{D}_{\mathrm{eff}} \Delta \Delta \bar{w}=\bar{q}_{n}-\frac{\bar{D}_{\mathrm{eff}}}{\bar{\Gamma}} \Delta \bar{q}_{n}
$$


where $\bar{D}_{\text {eff }}=\bar{C}_{22}+\bar{C}_{33}$ is the Laplace transform of the effective bending stiffness relaxation function, $\bar{w}=\overline{\boldsymbol{u}} \cdot n$ is the Laplace transform of the plate deflection, $\bar{q}_{n}=\bar{q} \cdot n$ is Laplace transform of the transverse load, respectively. Note, that here $\bar{D}_{\text {eff }}=D_{\text {eff }}^{0} \bar{\mu}(s)$, where $D_{\text {eff }}^{0}=\left(C_{22}+C_{33}\right) / \mu(t)$.

To analyze the influence of the transverse shear stiffness on the deflection of the plate, let us consider the bending of a rectangular plate made of a functionally graded material. Let us assume that $x_{1} \in[0, a], x_{2} \in[0, b]$, where $a$ and $b$ are the length and the width of the plate, respectively. Using the assumption that $\nu=$ const and the Eqs. (14), (15), and (24) are valid, we can rewrite Eq. (32) in the following form

$$
\bar{D}_{\text {eff }} \Delta \Delta \bar{w}=\bar{q}_{n}-\frac{2}{1-\nu} \frac{1}{\lambda^{2} h^{2}} \Delta \bar{q}_{n} .
$$

Introducing dimensionless variables by the formulas

$$
W=h^{-1} w, \quad X_{1}=h^{-1} x_{1}, \quad X_{2}=h^{-1} x_{2}, \quad X_{1} \in\left[0, \frac{a}{h}\right], \quad X_{2} \in\left[0, \frac{b}{h}\right],
$$

Eq. (33) transforms to

$$
\bar{\mu}(s) \Delta \Delta \bar{W}=Q-\frac{2}{1-\nu} \frac{1}{\lambda^{2} h^{2}} \Delta Q .
$$

Here

$$
\Delta=\frac{\partial^{2}}{\partial X_{1}^{2}}+\frac{\partial^{2}}{\partial X_{2}^{2}}, \quad Q=\frac{\bar{q}_{n} h^{3}}{D_{\mathrm{eff}}^{0}} .
$$

Let us consider a sinusoidal load

$$
q_{n}=Q_{0}(t) \sin \frac{\pi h X_{1}}{a} \sin \frac{\pi h X_{2}}{b}
$$

and the boundary conditions (3). Then

$$
Q=\bar{Q}_{0}(s) \sin \frac{\pi h X_{1}}{a} \sin \frac{\pi h X_{2}}{b},
$$

and the solution of Eq. (34) is given by

$$
\bar{W}=\frac{K}{\eta^{2}} \frac{\bar{Q}_{0}(s)}{\mu(s)} \sin \frac{\pi h \bar{x}_{1}}{a} \sin \frac{\pi h \bar{x}_{2}}{b}, \quad K=1+\frac{2 \eta}{1-\nu} \frac{1}{\lambda^{2} h^{2}}, \quad \eta=\left(\frac{\pi h}{a}\right)^{2}+\left(\frac{\pi h}{b}\right)^{2} .
$$

For the Kirchhoff's plate theory one gets

$$
K=K_{K} \equiv 1,
$$

for the homogeneous plate modeled in the sense of Mindlin's plate theory

$$
K=K_{M} \equiv 1+\frac{2 \eta}{1-\nu} \frac{1}{\pi^{2}},
$$

and for the FGM plate

$$
1+\frac{2 \eta}{1-\nu} \frac{L^{2} m_{\min }^{2}}{\pi^{2} h^{2}} \leq K \leq 1+\frac{2 \eta}{1-\nu} \frac{L^{2} m_{\max }^{2}}{\pi^{2} h^{2}} .
$$

The influence of the shear stiffness on the deflection of the elastic FGM plate was given in [21]. For the viscoelastic plate both the qualitative and the quantitative influence of the shear stiffness is the same as in [21].

For example, let us consider an open-cell foam and the following values $\nu=0.3, a=b, h=0.05 a, \alpha=0.9$. Using the calculation in [21] we obtain the following values of $\lambda: \lambda=0.83 / h$ for $n=2, \lambda=0.82 / h$ for $n=5$. The corresponding values of the factor $K$ are given by

$$
K_{\mathrm{M}} \approx 1.014, \quad K \approx 1.20 \quad(n=2), \quad K \approx 1.21 \quad(n=5) .
$$

That means that for the functionally graded plates the influence of the transverse shear stiffness may be significant. As well as for elastic FGM plates for other types of boundary conditions, the influence on the deflection may be greater than for the used simple-support type boundary conditions. 


\section{Discussion and outlook}

The considered concept to model FGM plates within the framework of a 5-parametric theory of plates applying the direct approach has an advantage in comparison with the classical theories of sandwich or laminated plates. The reason for this conclusion is that the suggested model is not based on a priori hypotheses about the stress, strain, or displacement states in the plate. Such an on hypotheses based model yields to a good agreement with experimental results if the plate is composed of classical structural materials. Since the foams are materials with a very complex microstructure, simple hypotheses cannot be established and a hypotheses-free theory results in better predictions of the global mechanical behavior. The main conclusion from the results presented here is that assuming linear viscoelastic behavior one gets similar improvements of the predictions like in the elastic case. This result follows immediately from the application of the Boltzmann's correspondence principle and the Laplace transform. Further investigations should be directed on more complex constitutive equations of viscoelastic solids $(\nu \neq$ const, non-isotropic case, non-symmetric material properties with respect to the mid-plane, thermomechanical behavior) and the description of the creep phenomenon in plates made of metal foams.

Acknowledgements The research work was partially supported by the Russian Foundation of Basic Research under grant 07-0813589-ofi_c. In addition, the authors want to acknowledge the reviewers for the stimulating reviews.

\section{References}

[1] P. Haupt, Continuum Mechanics and Theory of Materials, 2nd edition (Springer, Berlin, 2002).

[2] M. F. Ashby, A. G. Evans, N. A. Fleck, L. J. Gibson, J. W. Hutchinson, and H. N. G. Wadley, Metal foams: a Design Guide (Butterworth-Heinemann, Boston, 2000).

[3] J. Banhart, M. F. Ashby, and N. A. Fleck (eds.), Metal foams and porous metal structures (MIT Publishing, Bremen, 1999).

[4] H. P. Degischer and B. Kriszt (eds.), Handbook of Cellular Metals. Production, Processing, Applications (Wiley-VCH, Weinheim, 2002).

[5] L. J. Gibson and M. F. Ashby, Cellular Solids: Structure and Properties, 2nd edition (Cambridge University Press, Cambridge, 1997).

[6] N. Mills, Polymer Foams Handbook. Engineering and Biomechanics Applications and Design Guide (Butterworth-Heinemann, Boston, 2007).

[7] A. Kraatz, Berechnung des mechanischen Verhaltens von geschlossenzelligen Schaumstoffen unter Einbeziehung der Mikrostruktur, Diss., Zentrum für Ingenieurwissenschaften, Martin-Luther-Universität Halle-Wittenberg, Halle, 2007.

[8] R. Kienzler, Archive of Applied Mechanics (Ingenieur-Archiv) 72(4-5), 229-247 (2002).

[9] J. Kaplunov, E. Kossovich, and E. Nolde, Dynamics of thin walled elastic bodies (Academic Press, San Diego, 1998).

[10] R. Kienzler, H. Altenbach, and I. Ott (eds.), Critical Review of the Theories of Plates and Shells, New Applicatitions, Lect. Notes Appl. Comp. Mech., Vol. 16, Berlin, 2004, Springer.

[11] P. M. Naghdi, The theory of plates and shells, in: Handbuch der Physik, edited by S. Flügge, Festkörpermechanik Vol. VIa/2 (Springer, Berlin, 1972), pp. 425-640.

[12] E. Reissner, Appl. Mech. Rev. 38(11), 1453-1464 (1985).

[13] P. A. Zhilin, Int. J. Solids Struct. 12, 635-648 (1976).

[14] H. Altenbach and P. A. Zhilin, Uspekhi Mekhaniki 11(4), 107-148 (1988).

[15] H. Altenbach, Ingenieur-Archiv 58, 215-228 (1988).

[16] H. Altenbach and P. A. Zhilin, The theory of simple elastic shells, in: Critical review of the theories of plates and shells and new applications, edited by R. Kienzler, H. Altenbach, and I. Ott, Lect. Notes Appl. Comp. Mech. Vol. 16 (Springer, Berlin, 2004), pp. 1-12.

[17] P. A. Zhilin, Applied mechanics. Foundations of the Theory of Shells (in Russ.) (St. Petersburg State Polytechnical University, St. Petersburg, 2006).

[18] H. Altenbach, Mech. Solids 22(1), 135-141 (1987).

[19] H. Altenbach, Int. J. Solids Struct. 37(25), 3503-3520 (2000).

[20] H. Altenbach, ZAMP 51, 629-649 (2000).

[21] H. Altenbach and V. A. Eremeyev, Arch. Appl. Mech. pp. 1-18, DOI10.1007/s00419-007-0192-3 (2007).

[22] A. Lurie, Theory of Elasticity, Foundations of Engineering Mechanics (Springer, Berlin, 2005).

[23] R. M. Christensen, Theory of Viscoelasticity. An Introduction (Academic Press, New York, 1971).

[24] S. P. Timoshenko, Phil. Mag. 41(Ser. 6), 744-746 (1921).

[25] R. D. Mindlin, Phil. Mag. 18, 31-38 (1951).

[26] E. Reissner, J. Math. Phys. 23, 184-194 (1944).

[27] J. Stoer and R. Bulirsch, Introduction to Numerical Analysis (Springer, New York, 1980).

[28] P. Hartman, Ordinary Differential Equations (Wiley, New York, 1964).

[29] L. Collatz, Eigenwertaufgaben mit Technischen Anwendungen (Akademische Verlagsgesellschaft, Leipzig, 1963). 